\title{
Lysine malonylome may affect the central metabolism and erythromycin biosynthesis pathway in Saccharopolyspora erythraea
}

Jun-Yu Xu, Zhen Xu, Ying Zhou, and Bang-Ce Ye*

Lab of Biosystems and Microanalysis, State Key Laboratory of Bioreactor

Engineering, East China University of Science and Technology, Shanghai, 200237,

China.

\section{Supporting Information List}

Figure S-1 :INTERPRO domain analysis for lysine malonylated proteins in $S$. erythraea.

Figure S-2 : Parameters of network of malonylated protein in Figure 6 and Figure 7. Analysis for networks of malonylated proteins using Cytoscape (2.8.2) software and STRING database.

Figure S-3 : Parameters of MCODE tool for clusters of malonylated protein in Figure 7 (A)-(D).

Figure S-4 : The details of 4 clusters with high score by using cytoscope.

Figure S-5 : Overview of the phosphorylated peptides enrichment results: (A). Overview of the phosphorylated peptides enrichment used in this study. (B). Distribution of singly, doubly and triply phosphorylated peptides. (C). Pie chart representation of identified phosphorylation site distribution. 
Figure S-6 : All 192 malonylated spectras and all 220 phosphorylated spectras, together with all 158 malonylated spectras in the quantitative malonylome analysis identified in this study.

Table S-1 : List of all 192 identified Kmal peptides identified in the present study.

Table S-2 : List of all 220 identified phosphorylated peptides identified by MaxQuant software.

Table S-3 : List of protein quantification results in the stable isotope dimethyl labeling S. erythraea samples.

Table S-4 : List of sites quantifiable for Kmal in the two growth state in S. erythraea. Table S-5 : Gene Ontology (GO) analysis of lysine-malonylated proteins. A. Malonylation-enriched biological processes (BP). B. Malonylation-enriched cellular compartments (CC). C. Malonylation-enriched molecular functions (MF).

Table S-6 : KEGG pathway enrichment analysis of lysine-malonylated proteins.

Table S-7 : Enrichment analysis for INTERPRO domains of lysine-malonylated proteins.

Table S-8 : Motif analysis for lysine-malonylated proteins.

Table S-9 : Protein interaction networks of malonylated proteins using the STRING database by MCODE. The top 4 MCODE networks are listed.

Table S-10 : List of primers used in the study. 
Figure S-1 : INTERPRO domain analysis for lysine malonylated proteins in $S$. erythraea.
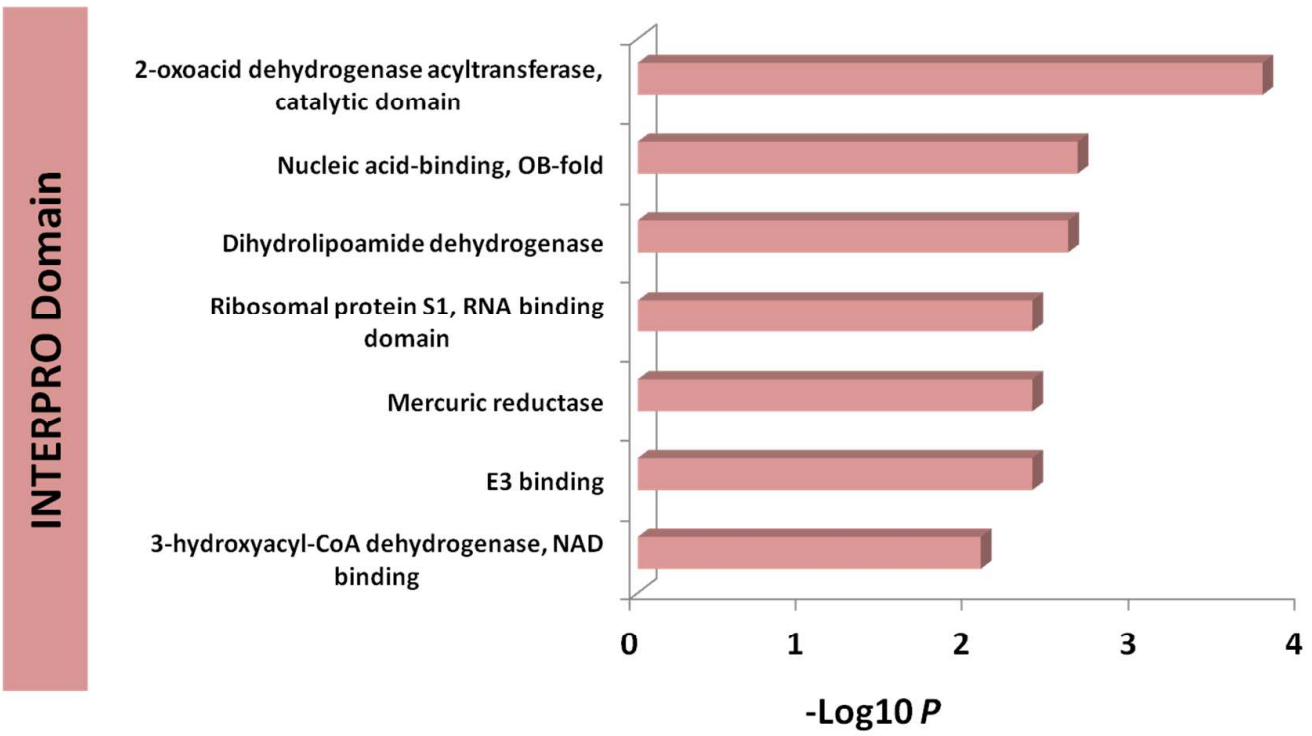

Figure S-2 : Parameters of network of malonylated protein in Figure 6 and Figure 7.

Analysis for networks of malonylated proteins using Cytoscape (2.8.2) software and STRING database.

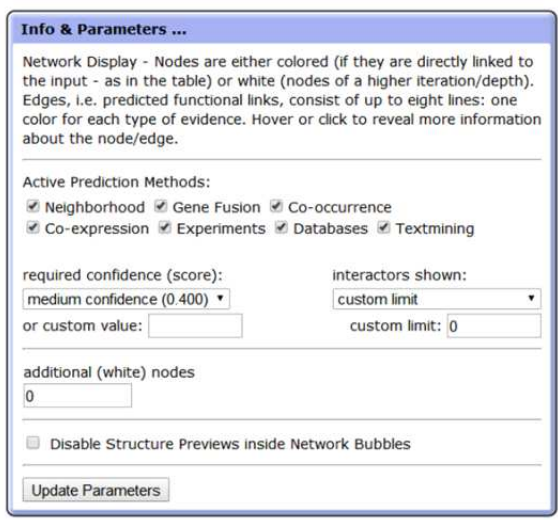

Description: Loading Network

Status:

Successfully loaded network from: xml_summary.U_lhMowRWAqY.xml

Network contains 109 nodes and 3,893 edges

Network is under 10000 nodes. A view has been created automatically. 
Figure S-3 : Parameters of MCODE tool for clusters of malonylated protein in Figure 7 (A)-(D).

\begin{tabular}{|c|c|c|c|}
\hline Edit or & Filters & $\triangle M C O D$ & $D_{4} \mid r$ \\
\hline \multicolumn{4}{|c|}{$\begin{array}{l}\text { Find Cluster (s) } \\
\text { (- in Whole Iletwork } \\
\text { from Selection }\end{array}$} \\
\hline \multicolumn{4}{|c|}{ Advanced Options $\mathbf{T}$} \\
\hline \multicolumn{3}{|c|}{ Include Loops } & $\square$ \\
\hline \multicolumn{3}{|c|}{ Degree Cutoff } & 2 \\
\hline \multicolumn{4}{|c|}{ Cluster Finding } \\
\hline \multicolumn{3}{|c|}{ Haircut } & 目 \\
\hline \multicolumn{3}{|c|}{ Fluff } & 回 \\
\hline \multicolumn{3}{|c|}{ Ilode Score Cutoff } & 0.2 \\
\hline \multicolumn{3}{|c|}{$\mathrm{K}$-Core } & 2 \\
\hline \multicolumn{3}{|c|}{ Max. Depth } & 100 \\
\hline
\end{tabular}

Figure S-4 : The details of 4 clusters with high score by using cytoscope.

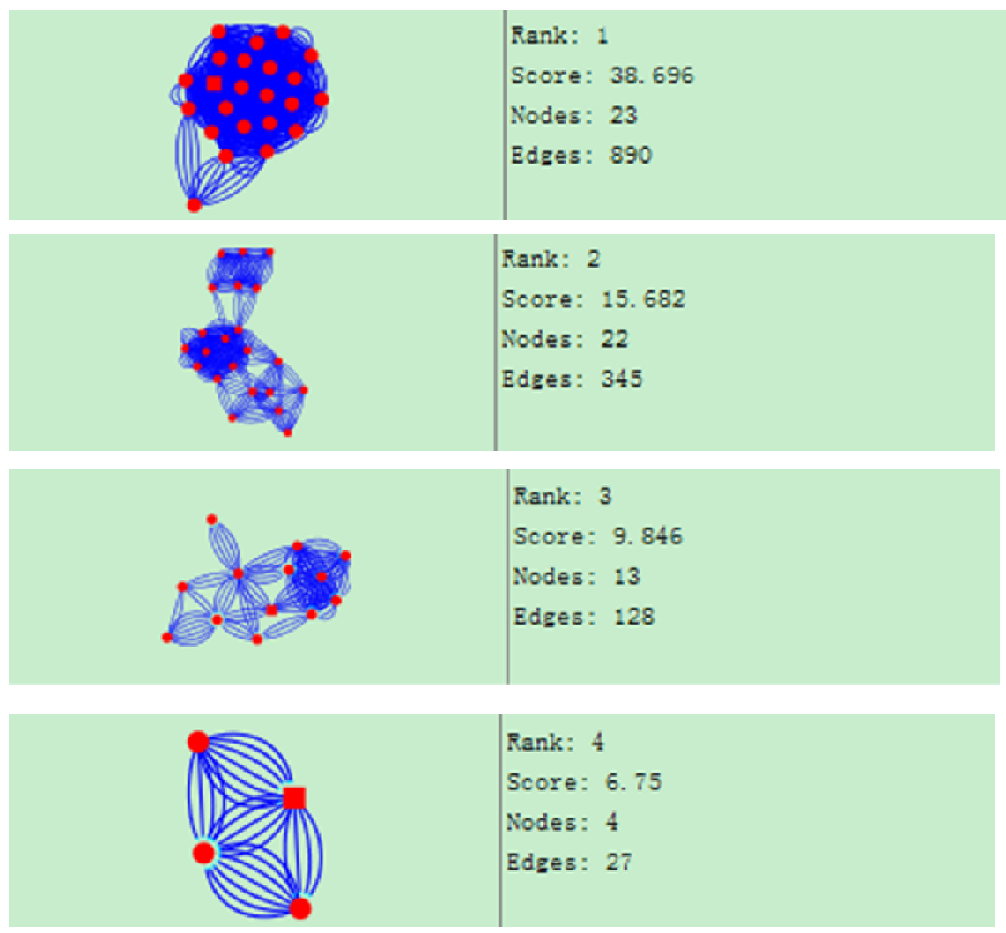


Figure S-5 : Overview of the phosphorylated peptides enrichment results: (A). Overview of the phosphorylated peptides enrichment used in this study. (B). Distribution of singly, doubly and triply phosphorylated peptides. (C). Pie chart representation of identified phosphorylation site distribution.

A

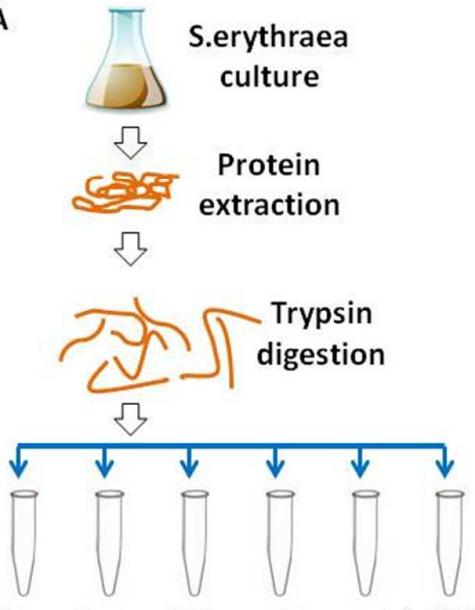

Phosphopeptide enrichment (TiO2) \3

Orbitrap Fusion mass Spectrometer analysis

\3.

Data analysis/ Bioinformatics
B
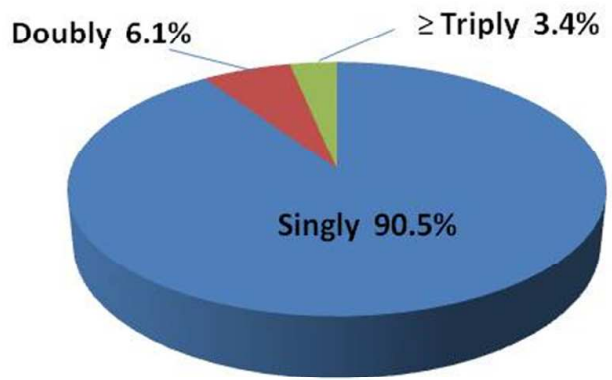

C

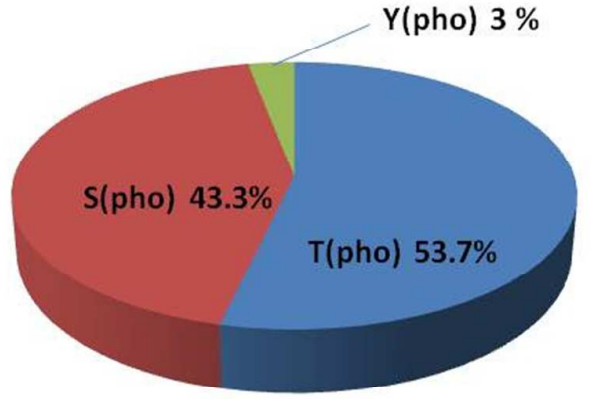

